\title{
Absence of vortex lattice melting in a high-purity $\mathrm{Nb}$ superconductor
}

\author{
C. J. Bowell,,${ }^{1} *$ R. J. Lycett, ${ }^{1}$ M. Laver, ${ }^{2}$ C. D. Dewhurst,${ }^{3}$ R. Cubitt,${ }^{3}$ and E. M. Forgan ${ }^{1}$ \\ ${ }^{1}$ School of Physics and Astronomy, The University of Birmingham, Birmingham B15 2TT, United Kingdom \\ ${ }^{2}$ Laboratory for Neutron Scattering, Paul Scherrer Institut, 5232 Villigen PSI, Switzerland \\ ${ }^{3}$ Institut Laue Langevin, BP 156, F-38042 Grenoble, France
}

(Received 2 June 2010; revised manuscript received 20 September 2010; published 11 October 2010)

\begin{abstract}
The state of the vortex lattice extremely close to the superconducting to normal transition in an applied magnetic field is investigated in high-purity niobium. We observe that thermal fluctuations of the order parameter broaden the superconducting to normal transition into a crossover but no sign of a first-order vortex lattice melting transition is detected in measurements of the heat capacity or the small-angle neutron scattering (SANS) intensity. Direct observation of the vortices via SANS always finds a well-ordered vortex lattice. The fluctuation broadening is considered in terms of the lowest Landau-level theory of critical fluctuations and scaling is found to occur over a large $H_{c 2}(T)$ range.
\end{abstract}

DOI: 10.1103/PhysRevB.82.144508

PACS number(s): 74.25.Uv, 61.05.fg, 74.25.Bt, 74.70.Ad

\section{INTRODUCTION}

Thermal fluctuations are large in the high- $T_{c}$ superconductors and their signature can be seen over a significant region of the superconducting phase diagram. In high-quality samples, the effects of disorder can be negligible compared with thermal fluctuations and a clear first-order transition, associated with melting of the vortex lattice ${ }^{1,2}$ occurs well below the "mean-field" superconducting to normal transition temperature, $T_{c 2}(H)$. However, in a low- $T_{c}$ sample, thermal fluctuations are significantly smaller due to the low temperature, the high vortex rigidity, and strong intervortex forces. Using the Lindemann criterion (that the lattice melts when fluctuations reach a critical fraction of the lattice spacing), vortex lattice melting is predicted ${ }^{3}$ to occur only several millikelvin below $T_{c 2}(H)$.

The small thermal energy in low- $T_{c}$ superconductors makes pinning of the vortex lattice much more significant, ${ }^{4}$ hence phase transitions related to this pinning become an alternative theoretical scenario. ${ }^{5,6}$ For example, a disordering transition in the vortex lattice of niobium has been observed ${ }^{7}$ directly by small-angle neutron scattering (SANS) and is associated with the peak effect ${ }^{8}$ [increased pinning of the vortices, linked to a softening of the vortex lattice below $\left.T_{c 2}(H)\right]$. The position of this transition varies with niobium sample purity ${ }^{9}$ and is not observed, to within $20 \mathrm{mK}$ of $T_{c 2}(H)$, in a high-purity sample. ${ }^{10}$ Heat-capacity measurements on $\mathrm{Nb}_{3} \mathrm{Sn}$ (Ref. 11) show signs of a vortex lattice phase transition in a sample with significant vortex lattice pinning (and showing the peak effect), hence it is not clear whether this transition is thermal melting or pinninginfluenced disordering. To investigate thermal melting of the vortex lattice, a high-quality low-pinning sample is clearly important. Here we present heat-capacity and SANS measurements on high-purity niobium. We observe the effects of thermal fluctuations of the superconducting order parameter but find no evidence for either a first-order melting transition or for order-disorder transitions associated with vortex pinning.

\section{METHODS}

Heat-capacity measurements were performed using the ac technique of Sullivan and Seidel. ${ }^{12}$ The sample is strongly thermally connected to a thermometer and heater, and weakly connected to a bath. An ac of frequency $\frac{1}{2} \omega$ is passed through the heater so that heat is generated at a rate $\frac{1}{2} P_{0}[\cos (\omega t)+1]$. Conditions are such that: (a) the heat capacities of the thermometer and heater are much less than that of the sample. (b) The sample heater and thermometer come into equilibrium with a time constant much less than the inverse of the angular frequency, $\omega$. (c) The sample to bath relaxation time is much longer than the inverse frequency. Under these conditions, the temperature of the sample is given by a dc increase above the temperature of the bath, plus an ac component,

$$
T_{\mathrm{ac}}=P_{0} \cos (\omega t) /(2 \omega C),
$$

where $C$ is the heat capacity of the sample (plus negligible contributions from heater and thermometer). The heat capacity can then be measured by accurate measurement of the size of the ac temperature oscillation. One important caveat of using this technique is that changes in the heat capacity over a range equal to the amplitude of the temperature oscillation are smoothed over. It is therefore important to set the size of the temperature oscillation to be small enough that sufficiently detailed changes in the heat capacity can be resolved. The size of the ac temperature oscillation at the sample was therefore set at less than $1 \mathrm{mK}$ (typically 0.2 $\mathrm{mK})$.

A schematic of the cryostat insert is shown in Fig. 1. A $14 \mathrm{~g}$ niobium sample (Nb1) was attached to a copper holder by nylon thread, and thermally connected to it by copper wire to give a thermal time constant of $\sim 3 \mathrm{~s}$ to the holder. Heat was applied to the sample by passing a current at $4 \mathrm{~Hz}$ along a small amount of resistive wire wound noninductively around the sample. Measurement of the oscillatory part of the sample temperature was performed using a ground-down GaAlAs diode; the ac component of the voltage across it was measured using a lock-in-amplifier (LIA) at $8 \mathrm{~Hz}$. The sample holder consisted of a large block of copper to act as a low-pass thermal filter. The sample holder was strongly thermally connected to a temperature control plate, where the base temperature of the sample was controlled above that of 



FIG. 1. (Color online) Schematic of the cryostat insert for the heat-capacity measurements showing how the sample holder connects to the $1 \mathrm{~K}$ Pot via the temperature control plate. All of these were mounted inside a vacuum can, with no exchange gas. Heaters and thermometers were present on the sample, on the temperature control plate and on the $1 \mathrm{~K}$ Pot.

the $1 \mathrm{~K}$ Pot during a measurement scan. The required temperature stability $(\sim 20 \mu \mathrm{K})$ was achieved using a homemade analog PID (proportional, integral, and derivative) feedback system that heated the plate. The sensor was a high-sensitivity carbon resistance thermometer attached to the temperature control plate, with its resistance measured by an audio-frequency ac bridge with a LIA as null detector. Fine temperature scans were made by adding a ramped dc voltage to the LIA dc output to shift the control point of the PID controller. Absolute measurement of the temperature at the temperature control plate was achieved by a calibrated Ge thermometer mounted onto the plate (but away from the applied magnetic field). The sample temperature was corrected to take into account the dc component of the heating at the sample.

During a measurement, the temperature was ramped with very small steps through the superconducting transition at fixed field both on warming and on cooling. Normal-state measurements were taken $20 \mathrm{mT}$ higher in field. A typical scan took $24 \mathrm{~h}$. A measurement of the amplitude of the ac voltage from the GaAlAs thermometer was typically taken every $300 \mathrm{~s}$, with the lock-in-amplifier averaging the signal with a time constant of typically $100 \mathrm{~s}$. This averaging introduced a lag between warming and cooling measurements which has been corrected for in the measurements presented in the paper.

The sample was aligned in the center of a magnetic field with uniformity three parts in $10^{4}$ over the volume of the sample. Two small superconducting coils were arranged either side of the sample to allow an optional ac field of amplitude $0.7 \mathrm{G}$ to be applied perpendicular to the main field.

SANS measurements were performed on the instrument D22 at the Institut Laue Langevin, Grenoble but with the instrument temperature control enhanced to achieve the required ultrafine temperature control. While the external electronics were the same as the heat-capacity measurements, the nature of neutron cryostats required a different setup in the cryostat insert. There was a low pressure of helium exchange gas in the sample chamber and its temperature was set by the relatively coarse temperature control of the instrument. The sample was mounted in a copper can inside the sample chamber; the can temperature was raised above that of the instrument control using a heater and carbon resistance thermometer attached inside the can, and PID feedback was used, as in the heat-capacity measurements to perform tem-

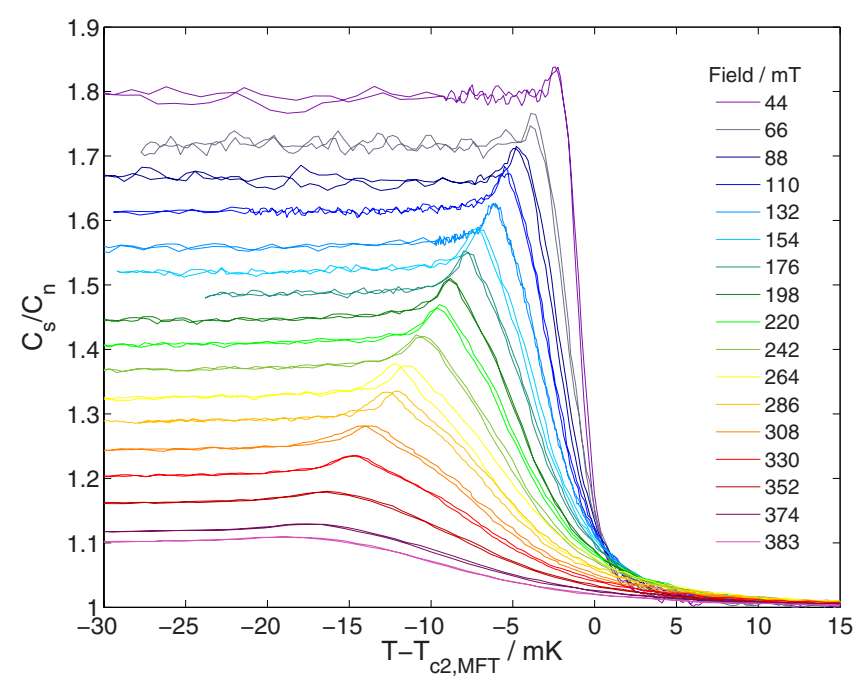

FIG. 2. (Color online) The ratio of the superconducting and normal-state heat capacities, $C_{s} / C_{n}$, measured as a function of temperature in the niobium sample $\mathrm{Nb}$. The magnetic field was applied parallel to the [111] crystal axis. Normal-state measurements, $C_{n}$, were taken $20 \mathrm{mT}$ higher in field. The temperature has been plotted as $T-T_{c 2, M F T}$, where $T_{c 2, M F T}(H)$ is the value the transition temperature would have in the absence of fluctuations and is taken as the temperature at which the fluctuation heat capacity is a fraction, 0.2 , of the mean-field jump in $C$ (Ref. 18). When a small hysteresis is seen, measurements taken on warming show the higher transition temperature.

perature sweeps. The magnet that provided the main magnetic field was designed to give uniformity $1.5 \times 10^{-4}$ over a 24-mm-diameter spherical volume. As with the heat-capacity measurements, additional coils allowed a small ac field of amplitude $0.1 \mathrm{G}$ to be applied to the sample.

Our experiments required samples in which vortex lattice pinning was minimal. The $14 \mathrm{~g}$, barrel shaped, niobium sample (residual resistance ratio, $R R R=1000$ ) was made high purity and homogeneous by annealing in ultrahigh vacuum $\left(<3 \times 10^{-9}\right.$ torr $)$ for $48 \mathrm{~h}$ at $2300 \mathrm{~K}$. It was then oxygen treated $^{13}$ to reduce surface pinning. This sample was used in both the heat-capacity measurements and SANS measurements, and is known as Nb1. Two other samples (used in the SANS measurements), $\mathrm{Nb} 2$ and $\mathrm{Nb} 3$, were spherical, $11 \mathrm{~g}$ $(R R R=450)$ and ellipsoidal, $8 \mathrm{~g}(R R R=1000)$ respectively, and prepared in the same way.

\section{HEAT-CAPACITY MEASUREMENTS}

Measurements of the heat capacity of niobium were the first to show that critical fluctuations broaden $T_{c 2}(H)$ from a second-order phase transition into a crossover. ${ }^{14}$ While no first-order vortex lattice melting transition was observed in these measurements, they were performed at constant magnetic induction $B$ whereas constant magnetic field $H$ is required to observe a first-order transition clearly. Fig. 2 presents our measurements of the heat capacity of high-purity niobium over the full $H_{c 2}(T)$ range. The surface treatment given helps to make the sample almost perfectly magnetically reversible, to allow the measurements to be performed 

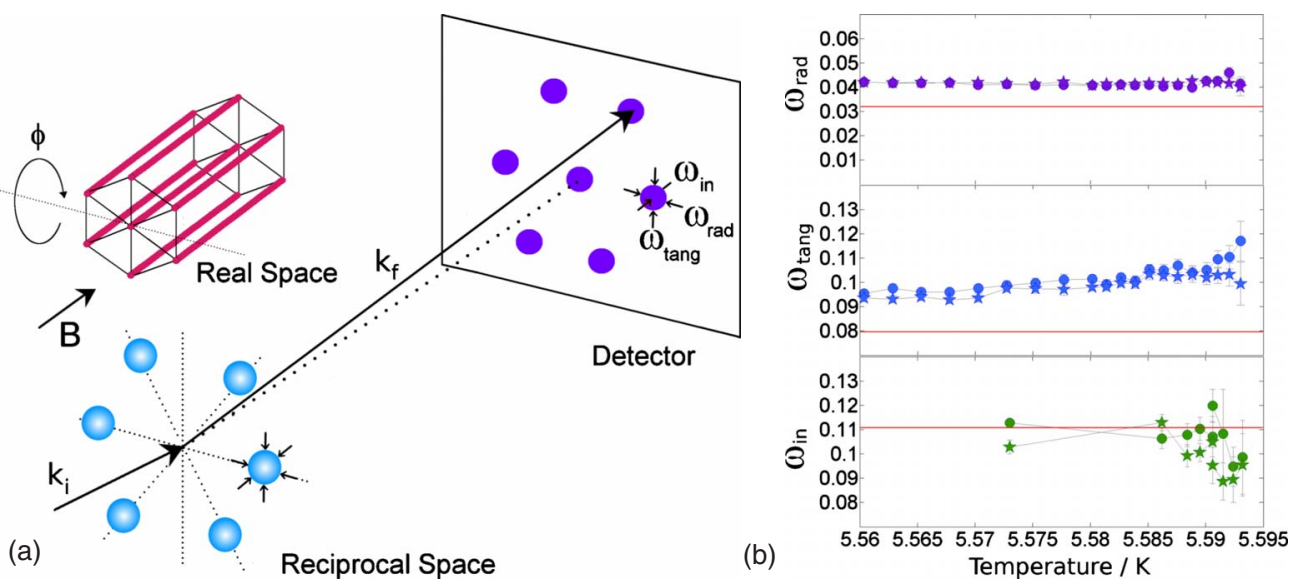

FIG. 3. (Color online) (a) Schematic to illustrate the geometry of a SANS experiment, where $\mathbf{k}_{i}$ and $\mathbf{k}_{f}$ represent the incident and scattered neutron beams, respectively. (b) The FWHM of Gaussian fits to the size of diffraction spots at the detector in the temperature regime of critical fluctuations at $200 \mathrm{mT}$ (applied parallel to the [111] axis of Nb1). Measurements were taken on cooling and are shown for all temperatures at which there was sufficient diffracted intensity to fit the spot shape. Backgrounds taken at $5.610 \mathrm{~K}$ were subtracted before fitting. For measurements of $\omega_{\text {rad }}$ and $\omega_{\text {tang }}, \phi$ was fixed at the angle that brought the top two first-order spots from the hexagonal lattice, left $(\bullet)$ and right $(\star)$, simultaneously onto the Bragg diffraction condition and maximized their intensity. Horizontal red lines mark the FWHM expected for a perfect lattice, where the width is due solely to instrument resolution.

at constant $H$. However, a small $(<0.5 \mathrm{mK})$ hysteresis can be seen at intermediate fields, resulting from some residual surface pinning of vortices and corresponding to flux trapping of less than $0.1 \mathrm{mT}$. Magnetization measurements on a smaller sample, similarly treated, showed no sign of the peak effect.

As shown in Fig. 2, we observe a transition broadened by critical fluctuations over the range $\sim 5$ to $30 \mathrm{mK}$ (depending on field), giving a peak below the mean-field transition and a long tail above it. At no temperature do we see sign of a first-order phase transition, of the type observed in the high- $T_{c}$ superconductors, which would appear as a spike in the heat capacity. In $\mathrm{YBa}_{2} \mathrm{Cu}_{3} \mathrm{O}_{7-\delta}$ the entropy of vortex lattice melting was found to be $0.4 k_{B}$ per vortex per superconducting layer. ${ }^{2}$ In comparing the latent heat in a layered system with that in isotropic niobium, we take the layer spacing to be equivalent to the coherence length, $\xi$. Using parameters suitable for our niobium sample we estimate (Appendix) that melting would cause a spike in the heat capacity of height $\Delta C_{\text {melt }} \sim 1 \mathrm{~mJ} \mathrm{~mol}^{-1} \mathrm{~K}^{-1}$ in a field of $300 \mathrm{mT}$ (for a transition width $\Delta T_{\text {melt }} \sim 2 \mathrm{mK}$ ); this is $\sim 10 \%$ of the mean field jump, $\Delta C$, at the superconducting to normal transition, which would be readily resolvable in our measurements but is clearly not seen.

\section{SANS MEASUREMENTS}

We can also search for a melting transition by examining the state of the vortex lattice using SANS. This technique was used in the observation of melting/decoupling of "pancake" vortices in the high- $T_{c}$ material $\mathrm{Bi}_{2.15} \mathrm{Sr}_{1.95} \mathrm{CaCu}_{2} \mathrm{O}_{8+x}$ (Ref. 15) but the low scattering intensity allowed no investigation of the nature of the disordered vortex state. The straightforward geometry of SANS allows the size of the diffraction spot on the detector to be related to correlation lengths of the vortex lattice. ${ }^{16}$ This idea is illustrated in Fig. 3(a). The width $\omega_{\text {in }}$ is obtained from a "rocking curve" of diffraction intensity as a function of angle when $\phi$, the angle between the applied magnetic field/sample [111] axis and the incident neutron beam, is rocked through the Bragg condition. On the peak of the rocking curve, $\omega_{\text {rad }}$ and $\omega_{\text {tang }}$ are the widths of the diffraction spot on the detector, parallel and perpendicular, respectively, to the relevant $q$ vector. The collimation and wavelength spread contribute to these widths but there are additional contributions due to the crystallinity of the vortex lattice in the sample. Mosaic spread (i.e., me-

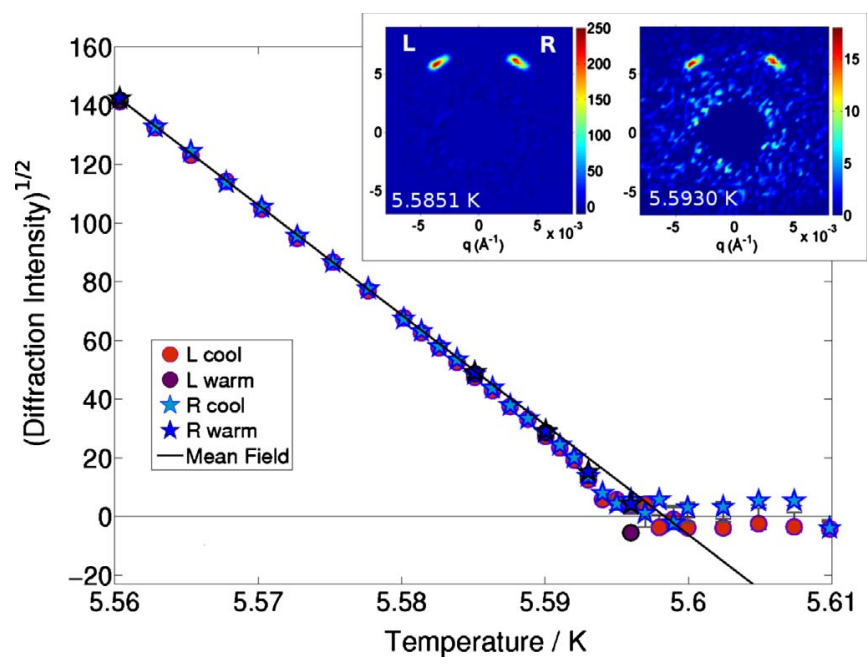

FIG. 4. (Color online) The square root of the diffracted intensity minus background, $\sqrt{I}\left(\propto\left|F_{h k}\right|\right)$, as a function of temperature in a field of $200 \mathrm{mT}$, measured during the same scan that $\omega_{\text {rad }}$ and $\omega_{\text {tang }}$ [Fig. 2(b)] were recorded. Negative values of $\sqrt{I}$ [calculated as $\operatorname{sgn}(I) \sqrt{|I|}]$ result from the background subtraction. The mean-field theory linear behavior is obtained from a fit to the temperature dependence outside the critical region. Insets show the diffraction pattern observed on the detector (with direct beam masked and background subtracted) for two temperature points. 

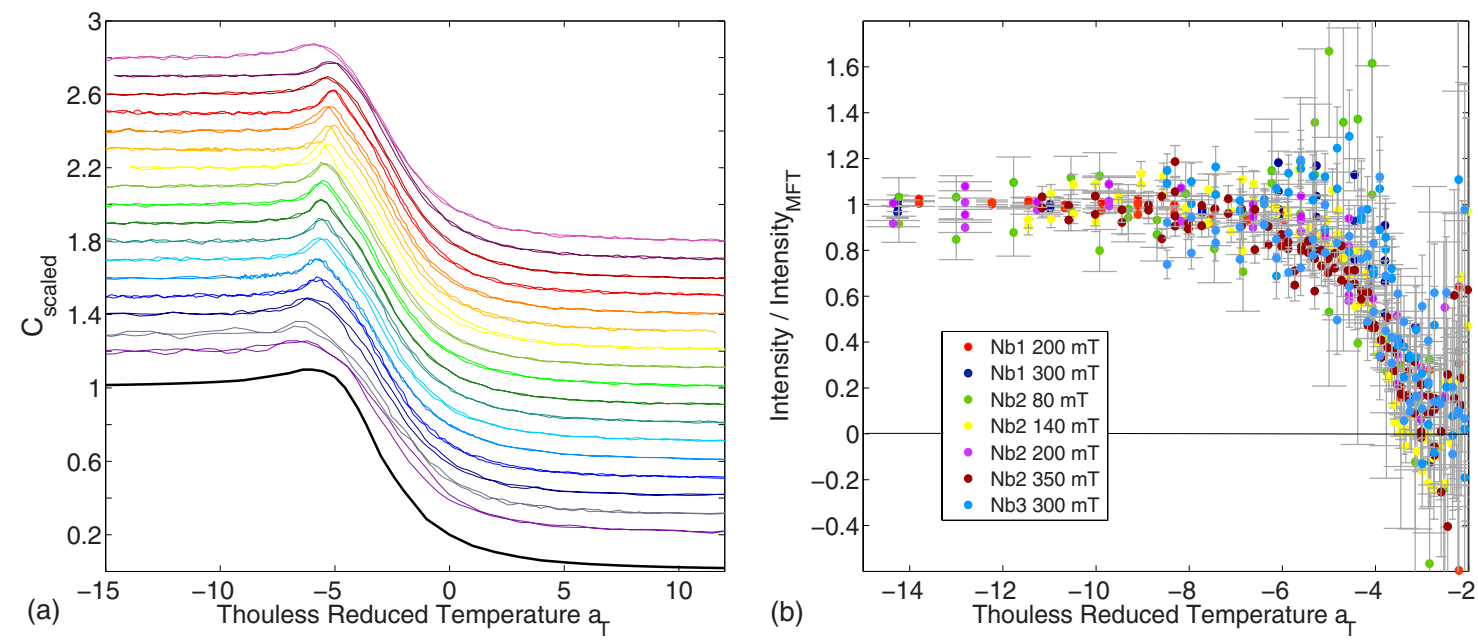

FIG. 5. (Color online) Scaling of the heat capacity and SANS intensity. (a) The heat-capacity measurements of Fig. 1, normalized to the mean-field behavior as $\left(C_{s}-C_{n}\right) /\left(C_{S, M F T}-C_{n}\right)$, plotted on the Thouless reduced temperature scale, $a_{T}$. $C_{S, M F T}$ was extrapolated through the transition from a linear fit of $C_{s} / C_{n}$ versus temperature below the fluctuation region (Ref. 14). The measurement at $44 \mathrm{mT}$ is shifted vertically by 0.2 units, to avoid overlap with the Thouless theoretical prediction (Ref. 18), which is plotted as the black line. All successive plots (at the same sequence of fields as in Fig. 1) are shifted by 0.1 units. (b) SANS intensity, normalized as $I / I_{M F T}$, where $I_{M F T}$ was found from a linear fit of $\sqrt{I}$ versus temperature below the critical region (as shown, for example, in Fig. 2) plotted versus the Thouless reduced temperature $a_{T}$. Measurements are shown for three samples, Nb1: barrel shaped, Nb2: spherical, and Nb3: ellipsoidal. All samples were prepared under similar condition with similar $R R R$ values. As no significant difference was observed between the left- and right-hand diffraction spots, or on warming and cooling, these measurements have been plotted with the same marker style for clarity. The error bars become large at high temperatures because the data points represent the ratio of two quantities, both of which are tending to zero.

andering of the average vortex line direction) is mainly seen in $\omega_{i n}$. Any spread in lattice spacing is more apparent in $\omega_{\text {rad }}$ while the spread in vortex lattice orientation (corresponding to rotations of the vortex lattice about the field direction) contributes to $\omega_{\text {tang }}$.

Measurements of these widths, on the same sample used in the heat-capacity measurements, $\mathrm{Nb1}$, are presented in Fig. 3(b). The perfection of the vortex lattice is such that the width of the rocking curve, $\omega_{i n}$, is limited by the instrument resolution while no changes in the radial spot width $\omega_{\text {rad }}$ are observed. There is a small increase in $\omega_{\text {tang }}$, the tangential width of the spot, but the $\sim 0.01^{\circ}$ change is extremely small compared with the spread that would be observed for an orientationally disordered vortex lattice, which would give a ring of diffracted intensity.

A signature of melting would also appear in the intensity, $I_{h k}$, of a diffraction spot, integrated over the rocking curve. This depends on the vortex lattice form factor, $F_{h k}$, which expresses the "field contrast" in the mixed state,

$$
I_{h k}=2 \pi \mu V\left(\frac{\gamma}{4}\right)^{2} \frac{\lambda_{n}^{2}}{\Phi_{0}^{2} q_{h k}}\left|F_{h k}\right|^{2},
$$

where $\mu$ is the incident neutron flux, $V$ is the sample volume, $\gamma$ is the neutron magnetic moment in nuclear magnetons, $\lambda_{n}$ is the neutron wavelength, and $\Phi_{0}$ is the flux quantum. Mean-field Ginzburg-Landau (GL) theory ${ }^{17}$ gives $F_{h k}$ proportional to the magnetization, $M$,

$$
F_{h k}=(-1)^{v} e^{-\pi v / \sqrt{3}} \mu_{0} M,
$$

where $v=h^{2}+h k+k^{2}$. In the absence of fluctuations the magnetization, and hence the form factor, should go linearly to zero as $T \rightarrow T_{c 2}(H)$. This linear variation in form factor with temperature has been verified to within $20 \mathrm{mK}$ of $T_{c 2}(H)$ on a high-purity niobium sample,${ }^{10}$ however that experiment did not have sufficient temperature resolution to probe the region where vortex lattice melting would be expected to occur. Measurement of the integrated intensity this close to $T_{c 2}(H)$ is only possible due to the relatively large field contrast in the mixed state of niobium. The equivalent experiment on a superconductor with a longer penetration depth would require unfeasibly long count times. ${ }^{15}$

As shown in Fig. 2(b), the rocking curve width, $\omega_{i n}$, for the vortex lattice in our sample is essentially independent of temperature in the critical region. Hence, apart from a proportionality constant, the temperature variation in $\left|F_{h k}\right|$ may be obtained by tilting the sample to the peak of the rocking curve and measuring diffraction intensity versus temperature with $\phi$ fixed. In the critical fluctuation region, we observe (Fig. 4) that the square root of the diffracted intensity, $\sqrt{I}$, falls below the linear mean-field theory prediction, but even in this region the lattice remains well ordered (inset Fig. 4). A first-order melting transition would appear as a discontinuity in the intensity, and this is not observed. It might be argued that temperature, field or sample inhomogeneity could provide the smearing of the SANS results. However, if we suppose that the measurements represent a smearing of mean-field linear variation in $\sqrt{I}$ with temperature near $T_{c 2}$ the intensity would lie above the mean-field extrapolation from lower temperatures, and not below it as we observe.

\section{CRITICAL SCALING}

To examine the origin of these deviations from mean-field theory, we look for a universal temperature scale which 

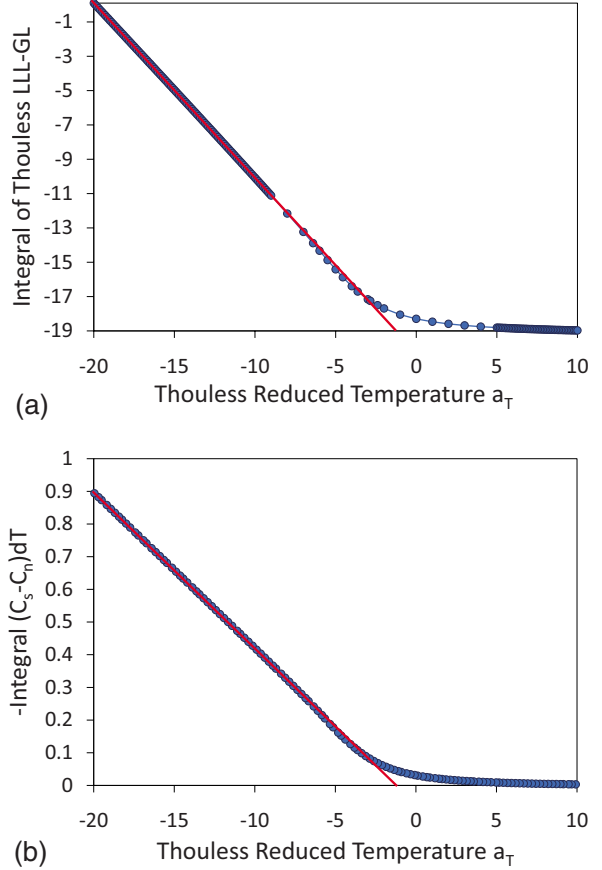

(b)

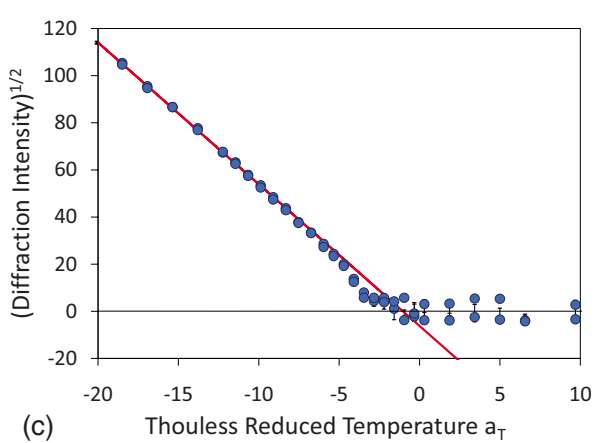

FIG. 6. (Color online) The magnetization extracted from the heat capacity and the SANS intensity. (a) $C_{s}-C_{n}$ from Thouless theory integrated with respect to temperature plotted versus the Thouless reduced temperature, $a_{T}$. (b) $C_{s}-C_{n}$ from experiment, integrated with respect to temperature and plotted versus $a_{T}$. Measurements taken from Nb1 in $198 \mathrm{mT}$. (c) The SANS diffraction intensity, presented as $\sqrt{I}(\propto M)$ versus $a_{T}$. Measurements taken from $\mathrm{Nb} 1$ in $200 \mathrm{mT}$. Red lines show the extrapolation of linear meanfield-theory behavior.

would allow measurements at different fields to collapse onto the same curve. Three-dimensional XY scaling is not suitable, as it requires a spatially uniform order parameter, which is not valid in niobium as the vortex cores are large. However, the lowest Landau-level GL (LLL-GL) theory of critical fluctuations is appropriate. The relevant temperature scale $a_{T}$, as calculated by Thouless, ${ }^{18}$ is given by $a_{T}$ $=\left(T-T_{c 2, M F T}\right) / \delta$, where

$$
\delta=\left(\frac{k_{B}}{8 \pi \xi_{0}^{3} \Delta C}\right)^{3 / 2}\left|\left(\frac{T}{B_{c 2}(0)}\right) \frac{d B_{c 2}(T)}{d T}\right|^{1 / 3}\left(\frac{B}{B_{c 2}(0)}\right)^{2 / 3} T .
$$

Critical scaling of the heat-capacity measurements, Fig. 5(a), is successful over a large region of the phase diagram, except at the highest and lowest fields. This confirms the fluctuation origin of the signal and rules out inhomogeneity broadening. In the field region where our measurements scale, we find that the long tail in heat capacity at high temperatures agrees well with the Thouless prediction within LLL-GL but the enhanced peak is narrower than expected from theory. This may demonstrate the need for higher-order terms in the fluctuations to be included in the theory, mixing of Landau levels at the transition, ${ }^{19}$ or a consequence of coupling between the vortex and crystal lattice (which also breaks the rotational degeneracy of the vortex lattice and gives it a preferred orientation).

As the neutron-scattering intensity originates from the magnetization, it would be of interest to see how its behavior compares to that of the heat capacity. The heat-capacity and SANS measurements were taken using different magnets, and different thermometers, neither of which had calibration accuracy-in absolute temperature-small compared with the widths of the transitions. However, by comparison of the data with theory, we have been able to intercompare data taken at different fields and temperatures - and data from the two different techniques - as a function of temperature difference from the mean-field transition temperature, $T_{c 2, M F T}$, even though there is no sharp transition once fluctuations are taken into account.

To calculate the magnetization of the sample from the heat capacity, we use the thermodynamic relation

$$
C_{s}-C_{n}=\mu_{0} T\left(\frac{d H_{c 2}}{d T}\right)\left(\frac{\partial M_{s}}{\partial T}\right)_{H} .
$$

Integrating the Thouless theoretical prediction for the heat capacity ${ }^{18}$ down from high temperatures, we obtain Fig. 6(a), which indicates that at temperatures below the critical region the magnetization varies linearly with temperature, which is the mean field GL result in the mixed state. However, it will be noticed that the magnetization extrapolates linearly to zero at a reduced temperature of $a_{T}=-1.0$. This indicates, as one would expect, that fluctuations not only broaden the transition but also suppress it below the mean-field value. In Fig. 6(b), experimental heat-capacity data is integrated in the same way and a similar graph is obtained. Finally, we consider the SANS data, where below the critical region the SANS intensity obeys the mean-field result $\sqrt{I} \propto M$. The SANS intensity measurements can be placed on the same temperature scale by taking the extrapolation of the meanfield behavior, where $\sqrt{I} \rightarrow 0$ at $a_{T}=-1.0$, as shown in Fig. 6(c).

We can now compare the temperature dependence of the magnetization obtained from these two different techniques. The magnetization derived from the heat-capacity measurements demonstrates superconducting fluctuations result in a long tail above $T_{c 2, M F T}$, giving in this region a diamagnetic magnetization larger than the mean-field extrapolation. However, the SANS intensity decreases faster than mean-field behavior and is already immeasurably small below $T_{c 2, M F T}$. The different temperature dependencies occurs because the heat-capacity measurements are sensitive to all fluctuations that give rise to an average magnetization while the SANS intensity is sensitive only to a spatially coherent contrast in 

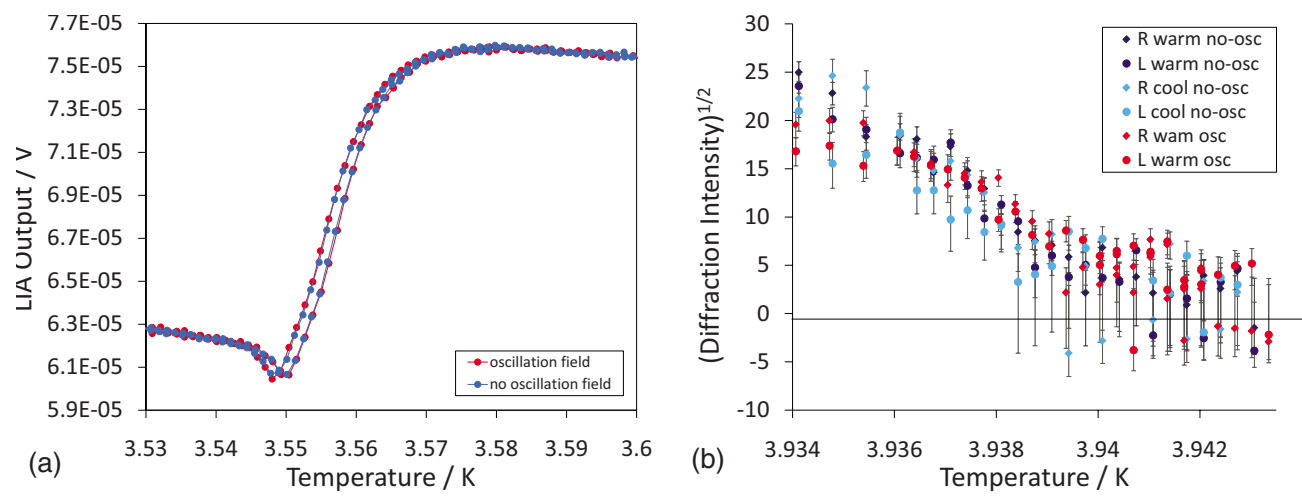

FIG. 7. (Color online) Investigation of a small oscillatory field applied in addition to the main field. (a) Raw LIA output $(\propto 1 / C)$ for Nb1 in a field of $308 \mathrm{mT}$. In one measurement, an oscillating field of amplitude $0.7 \mathrm{G}$, frequency $100 \mathrm{~Hz}$ was continually applied to the sample during the measurement. (b) Diffracted intensity from Nb3 taken with and without a small decaying oscillatory magnetic field applied to the sample before each measurement. The main field applied was $300 \mathrm{mT}$.

the magnetization. Fluctuations of the superconducting order parameter are expected to result in local-field fluctuations in a low- $\kappa$ superconductor such as niobium. ${ }^{18}$ Hence these local-field fluctuations reduce the spatially coherent magnetic field contrast, causing the decrease in SANS intensity below the mean-field value. This is confirmed in Fig. 5(b), where the SANS intensity in the critical region is shown to obey scaling once plotted on the $a_{T}$ temperature scale.

\section{OSCILLATING FIELD}

We also performed the heat-capacity and SANS measurements with a small oscillating magnetic field applied to the sample in addition to the main field. This type of field can help the vortices find their equilibrium position in the case of weak pinning. Fig. 7(a) shows the raw lock-in-amplifier output from the heat-capacity measurements (the signal is $\propto 1 / C$ and the output has not been corrected for time lag due to LIA time constant) taken under identical conditions except for the presence/absence of a small oscillatory magnetic field. The size of the magnetic field was too small to give a noticeable change in transition temperature in this scan (and because it was applied perpendicular, the value of the field was essentially unaltered; even parallel, $\Delta B_{c 2} \sim 0.7 \mathrm{G}$ corresponds to $\left.\Delta T_{c 2} \sim 0.2 \mathrm{mK}\right)$. The measurements with and without the oscillatory field are identical. Fig. 7(b) shows a similar experiment performed on the SANS measurements, except in this case the oscillatory field was applied between each measurement, and consisted of a decaying exponential oscillatory field, with initial amplitude $0.1 \mathrm{G}$. No difference in diffracted intensity between these two measurements can be observed above the noise. That we observed identical behavior, both with and without this additional oscillating field, illustrates the very low-pinning nature of our sample and confirms that all our measurements were performed on a vortex lattice in equilibrium.

\section{CONCLUSIONS}

In summary, measurements of the heat-capacity and SANS intensity in high-purity niobium show no sign of a first-order vortex lattice melting transition. Direct observation of the vortices by SANS always finds a well-ordered lattice. Fluctuations are observed to broaden the secondorder superconducting to normal transition into a crossover in both the heat-capacity and SANS intensity measurements. LLL scaling is successful over a large range of fields, characterizing the nature of the fluctuations. The fluctuation enhancement of the heat capacity is found to be more sharply peaked than the predictions of Thouless. ${ }^{18}$

We now have to consider why the vortex lattice does not melt in niobium, a task made difficult due to the paucity of theoretical work. Our measurements show that the temperature range over which thermal fluctuations affect the superconducting order parameter is in agreement with LLL theory. Calculations within LLL theory predict vortex lattice melting to occur at $a_{T}=-9.5$ but in a strongly type-II superconductor. ${ }^{20}$ This is well below our fluctuation region. How this prediction is modified for low $-\kappa$ niobium is a current theoretical challenge. One difference is that in the high- $T_{c}$ superconductors the melting temperature is well below the mean-field $T_{c 2}(H)$ so the order parameter is not changing much in magnitude with temperature, however, in niobium the melting temperature is expected to be very close to $T_{c 2}(H)$ and therefore the loss of phase coherence of the order parameter would occur in a region where it is rapidly changing.

The absence of melting may be due to interactions of the vortex lattice with the underlying crystal lattice, which is known to have a significant influence on vortex behavior at lower temperatures. For example, frustration between the ideal hexagonal vortex lattice and the cubic crystal lattice results in square, scalene, and isosceles vortex lattice structures when the field is applied along a [001] crystal axis of niobium. These structures have been confirmed to persist up to $H_{c 2}(T)$ to within $100 \mathrm{mK}$ of $T_{c 2}(H){ }^{21}$ These strong interactions with the crystal could maintain vortex lattice rigidity, preventing melting. It would be interesting to expand our measurements to applied field directions other than parallel to the [111] crystal axis, to examine the importance of this interaction in our fluctuation temperature regime.

A more unconventional explanation for the absence of vortex lattice melting in niobium is also a possibility. While 
there is no doubt that a first-order transition occurs in the high- $T_{c}$ superconductors, the microscopic details of the transition are still poorly understood, especially as direct observation of the vortices above the transition has not been achieved. Our results are compatible with the controversial proposal $^{22}$ that the vortex lattice is actually a liquid, but with a correlation length so large that it is experimentally indistinguishable from a solid, and the melting transition observed in the high- $T_{c}$ superconductors is related to decoupling of pancake layers and would not be expected to occur in our isotropic system.

\section{ACKNOWLEDGMENTS}

This work was supported by the UK EPSRC. Neutron experiments were performed on instrument D22 at the Institut Laue Langevin, Grenoble, France. We are grateful for discussions with M. A. Moore, B. Rosenstein, and B. Ya. Shapiro.

\section{ENTROPY OF MELTING}

Vortex lattice melting in $\mathrm{YBa}_{2} \mathrm{Cu}_{3} \mathrm{O}_{7-\delta}$ was accompanied by an entropy change of $\Delta S=0.4 k_{B}$ per vortex per layer. ${ }^{2} \mathrm{We}$ wish to make an estimate of the entropy change that would accompany vortex lattice melting in niobium. In comparing the latent heat in a layered system (where the vortex layer spacing is taken to be the distance between the $\mathrm{CuO}_{2}$ planes) with that in isotropic niobium, we take the layer spacing to be equivalent to the coherence length, so in niobium we expect $\Delta S=0.4 k_{B} /$ vortex $/ \xi$, with $\xi=29 \mathrm{~nm}$. (The work of Dodgson et al. ${ }^{23}$ suggests that the relevant length scale is the vortex spacing and not $\xi$; however their theory applies in the London limit, and in the case considered below there is only a factor $\sim 2$ difference between these two lengths). The entropy density, $\Delta s$, is related to the entropy per vortex per layer, $\Delta S$, by $\Delta S=d^{2} \xi \Delta s$, where $d$ is the intervortex spacing and $\xi$ is the coherence length/layer spacing. In a field of 300 $\mathrm{mT}\left[0.7 H_{c 2}(T=0)\right.$, giving $\left.T_{c 2}=3.5 \mathrm{~K}\right]$ the intervortex spacing is $d=7.7 \times 10^{-8} \mathrm{~m}$. This gives an entropy density of $\Delta s=32 \mathrm{~mJ} \mathrm{~m}^{-3} \mathrm{~K}^{-3}=3.48 \times 10^{-4} \mathrm{~mJ} \mathrm{~mol}^{-1} \mathrm{~K}^{-1}$.

We can make an estimate for the height of the heat capacity spike by assuming the spike is triangular in shape, with a transition width of $\Delta T_{\text {melt }}$. The width of this hypothetical melting transition will be related to sample homogeneity. In zero field (where fluctuations are only expected to be present on the scale of $\mathrm{nK}$ ) our $T_{c 2}(0)$ width of $0.4 \mathrm{mK}$ (measured from the heat capacity) is testament to the high quality of the sample. In a magnetic field we need to consider how much any variations in sample purity will broaden $T_{c 2}$, (additional to any broadening due to fluctuations of the order parameter). The upper critical field, $B_{c 2}$, depends on the GinzburgLandau parameter, $\kappa_{1}$, as $B_{c 2}(T)=\sqrt{2} \kappa_{1} B_{c}(T)$, and $\kappa_{1}$ depends on the electronic mean-free path as given ${ }^{24}$ by $\kappa=\kappa_{0}$ $+7500 \rho_{0} \sqrt{\gamma}$, where $\kappa_{0}$ is for the case of infinite mean-free path, $\rho_{0}$ is the residual resistivity and $\gamma$ is the Sommerfeld constant. Any variations in sample purity will give a spread in residual resistivity across the sample, resulting in a spread in upper critical field, $\Delta B_{c 2} \sim\left(\xi \Delta l / l^{2}\right) B_{c 2}$, where $l$ is the electron mean-free path. This estimation emphasizes the importance of using a high-purity sample.

We can relate ${ }^{25}$ electron mean-free path to the residual resistivity ratio, $R$, using $l=5 \times 10^{-9} R$, so for our sample with $R=1000, \quad l=200 \xi$. Therefore, using $\Delta T_{c 2}$ $\sim\left(\xi \Delta l / l_{2}\right) B_{c 2}\left(d B_{c 2} / d T\right)^{-1}$, we have, for an assumed inhomogeneity in $\Delta l$ of $5 \%$, a transition width due to inhomogeneity broadening of $\Delta T_{c 2} \sim 2 \mathrm{mK}$ in a field of $300 \mathrm{mT}$. If the width of the melting transition is the same: $\Delta T_{\text {melt }} \sim 2 \mathrm{mK}$, we can therefore estimate the height of the spike in the heat capacity to be $\Delta C_{\text {melt }} \sim 1.2 \mathrm{~mJ} \mathrm{~mol}^{-1} \mathrm{~K}^{-1}$ in a field of 300 $\mathrm{mT}$. The size of the mean-field heat-capacity jump in this field is $\Delta C_{M F T}=10 \mathrm{~mJ} \mathrm{~mol}^{-1} \mathrm{~K}^{-1}$ so the spike would be $12 \%$ of the jump.
*Present address: Department of Materials Science and Metallurgy, University of Cambridge CB2 3QZ, UK.

${ }^{1}$ E. Zeldov, D. Majer, M. Konczykowski, V. B. Geshkenbein, V. M. Vinokur, and H. Shtrikman, Nature (London) 375, 373 (1995).

${ }^{2}$ A. Schilling, R. A. Fisher, N. E. Phillips, U. Welp, D. Dasgupta, W. K. Kwok, and G. W. Crabtree, Nature (London) 382, 791 (1996).

${ }^{3}$ G. P. Mikitik and E. H. Brandt, Phys. Rev. Lett. 89, 259701 (2002).

${ }^{4}$ G. P. Mikitik and E. H. Brandt, Phys. Rev. B 68, 054509 (2003).

${ }^{5}$ G. P. Mikitik and E. H. Brandt, Phys. Rev. B 64, 184514 (2001).

${ }^{6}$ J. Kierfeld and V. Vinokur, Phys. Rev. B 61, R14928 (2000).

${ }^{7}$ P. L. Gammel, U. Yaron, A. P. Ramirez, D. J. Bishop, A. M. Chang, R. Ruel, L. N. Pfeiffer, E. Bucher, G. D'Anna, D. A. Huse, K. Mortensen, M. R. Eskildsen, and P. H. Kes, Phys. Rev. Lett. 80, 833 (1998).

${ }^{8}$ A. B. Pippard, Philos. Mag. 19, 217 (1969).
${ }^{9}$ X. S. Ling, S. R. Park, B. A. McClain, S. M. Choi, D. C. Dender, and J. W. Lynn, Phys. Rev. Lett. 86, 712 (2001).

${ }^{10}$ E. M. Forgan, S. J. Levett, P. G. Kealey, R. Cubitt, C. D. Dewhurst, and D. Fort, Phys. Rev. Lett. 88, 167003 (2002).

${ }^{11}$ R. Lortz, F. Lin, N. Musolino, Y. Wang, A. Junod, B. Rosenstein, and N. Toyota, Phys. Rev. B 74, 104502 (2006).

${ }^{12}$ P. F. Sullivan and G. Seidel, Phys. Rev. 173, 679 (1968).

${ }^{13}$ G. P. Van Der Mey, P. H. Kes, and D. De Klerk, Physica B \& C 95, 369 (1978).

${ }^{14}$ S. P. Farrant and C. E. Gough, Phys. Rev. Lett. 34, 943 (1975).

${ }^{15}$ R. Cubitt, E. M. Forgan, G. Yang, S. L. Lee, D. McK. Paul, H. A. Mook, M. Yethiraj, P. H. Kes, T. W. Li, A. A. Menovsky, Z. Tarnawski, and K. Mortensen, Nature (London) 365, 407 (1993).

${ }^{16}$ R. Cubitt, E. M. Forgan, D. Mck. Paul, S. L. Lee, J. S. Abell, H. Mook, and P. A. Timmins, Physica B 180-181, 377 (1992).

${ }^{17}$ E. H. Brandt, Rep. Prog. Phys. 58, 1465 (1995).

${ }^{18}$ D. J. Thouless, Phys. Rev. Lett. 34, 946 (1975). 
${ }^{19}$ Z. Tešanović and A. V. Andreev, Phys. Rev. B 49, 4064 (1994). ${ }^{20}$ D. Li and B. Rosenstein, Phys. Rev. B 65, 220504 (R) (2002).

${ }^{21}$ M. Laver, E. M. Forgan, S. P. Brown, D. Charalambous, D. Fort, C. Bowell, S. Ramos, R. J. Lycett, D. K. Christen, J. Kohlbrecher, C. D. Dewhurst, and R. Cubitt, Phys. Rev. Lett. 96, 167002 (2006).
${ }^{22}$ J. Yeo and M. A. Moore, Phys. Rev. B 64, 024514 (2001).

${ }^{23}$ M. J. W. Dodgson, V. B. Geshkenbein, H. Nordborg, and G. Blatter, Phys. Rev. B 57, 14498 (1998).

${ }^{24}$ B. B. Goodman, IBM J. Res. Dev. 6, 63 (1962).

${ }^{25}$ E. M. Forgan, Ph.D. thesis, University of Birmingham, 1970. 